\title{
New challenges in the automation of electric power systems
}

\begin{abstract}
An electric power system is composed by generating, transmission, distribution, and consumers subsystems. As consumption of electrical energy increases the operation of power systems becomes more complex, and more necessary is to have realtime monitoring and remote control of elements such as generators, substations, transmission lines and other physical facilities. This communication explores on new challenges in automation of operational processes originated by the increasing complexity of electric power systems. In particular the application of synchrophasor measurements to opportunately initiate automatic control actions under emergency conditions is briefly discussed.
\end{abstract}

Volume 4 Issue 2 - 2018

Patricio Robles

Pontificia Universidad Católica de Valparaíso, Chile

Correspondence: Patricio Robles, Pontificia Universidad Católica de Valparaíso, Chile, Tel 5632 2273696, Email patricio.robles@pucv.cl

Received: January 29, 2018 | Published: March 16, 2018

Keywords: transmission system, renewable energy, monitoring, voltaje, frequency, power stability, synchrophasor

Abbreviations: SIC, central interconnected system; SING, great north interconnected System; FACTS, flexible alternating current transmission systems; SVC, static var compensator; SCADA, supervisory control and data acquisition; WAMS, wide area measurements system; TCSC, thyristor-controlled series compensator

\section{Introduction}

Power system automation included monitoring, evaluation, analysis, and control of processes associated with generation and transmission of electric energy from power stations to customers. Three main processes are included: data acquisition, power system supervision and control, all working in a coordinated automatic fashion. Data acquisition refers to collect data in the form of measured analog current or voltages values or the status of devices (open or closed). Power system supervision is carried out through the acquired data either at a remote site or locally at the device site. Control refers to sending command messages to operate power system devices such as circuit breakers. In this communication these concepts are discussed in the context of the chilean electric sector considering the challenges originated by the recent interconnection of the two main electric system SIC and SING and new interconnection projected with other electrical systems. In November 2017 the two main electric systems of Chile, SIC and SING, have been interconnected by a 500 $\mathrm{kV}$ transmission system resulting in an interconnected grid with a geographical extension of about 3000 kilometers from south to north of the country. Concerned with the automation, it is expected that several operational challenges will arise due to this interconnection and additionally due to the sustained increase in the generation coming from renewable energies like eolic generators, geothermal and photovoltaic systems, contributing at present with about $20 \%$ of the produced energy. Traditionally the main issues related with the operation of Chilean electric systems have been those concerned with the control of the frequency and voltage for reaching stability of these variables. Power generation plants have systems that control the injected active and reactive power to the network and several
FACTS like SVC are operating in the Chilean $500 \mathrm{kV}$ transmission system. However the growth of this electrical system is leading to more requirements in data acquisition, data processing and control systems. Automation in the management of renewable energies is required considering that due to the variability in the available power, they introduce new uncertainties and parameters' variations into the power grid, so that automation systems are required to connect these generation systems to the network and inject the corresponding energy in a coordinated way.

A widely used tool for managing the operation of electric power systems is the SCADA which supervises controls, optimizes and manages generation and transmission systems. This system is used in the Chilean interconnected system and is combined with a WAMS based on synchrophasor measurements that allow simultaneous data acquisition from several points of the system in times of the order of milliseconds, enhancing the real-time knowledge of the operators about the state of the system. This makes possible to take corrective actions before the system undergoes a critical condition that could lead it to a blackout. ${ }^{2}$ For instance the simultaneous measurement of voltage phase angles $\theta_{i}(t)$ in distant substation buses and register of the Corresponding difference $\theta_{i j}(t)=\theta_{i}(t)-\theta_{j}(t)$ allow to opportunately initiate control actions such as generators redispatch ${ }^{3}$ or decrease power flows of heavily loaded transmission lines by the action of TCSC. ${ }^{4}$ A possible control strategy could be based in use of these measurements to construct the matrix $\mathrm{H}$ of dimension $N \cdot N$ with $\mathrm{N}$ being the number of buses of the grid and with elements $\theta_{i j}(t)$ and evaluate the Frobenius norm defined as $\llbracket H \rrbracket=\left(\sum_{i j} \theta_{i j}{ }^{2}\right)^{1 / 2}$. If this norm is higher than a reference value the beginning of a critical condition may be opportunately detected. Identifying the greater values of $\theta_{i j}$ the heaviest load circuits may be known in real time so that the corresponding control actions could be automatically taken.

\section{Conclusion}


As electrical power systems grow more requirements of robust control and automation schemes arise for obtaining a reliable operation. In this communication this issue has been briefly discussed in the context of the Chilean Interconnected System, in particular the application of synchrophasor measurements to opportunately initiate control actions in the case of heavily loaded transmission lines. For preventing blackouts produced by a cascade of events initiated by a local disturbance, it is advisable to make more research concerned with the automatic identification of the more convenient ways to separate the system in electric islands under a local disturbance and to get a balance between generation and load in each of these islands for keeping suitable values of the corresponding frequencies and voltages during the emergency condition.

\section{Acknowledgements}

This research Project was partially or fully sponsor by (Electrical Engineering Department, Pontificia Universidad Católica de Valparaíso).

\section{Conflict of interest}

The author declares there is no conflict of interest.

\section{References}

1. Joseph Richard, Nerey Mvungi. Concept of automation in management of electric power systems. International Journal of Electrical and Computer Engineering. 2014;8(12):1849-1853.

2. Hjörtur Jóhannsson, Markus Wache. System security assessment in realtime using synchrophasor measurements. Proceedings of 2013 IEEE PES Innovative Smart Grid Technologies; 2013 October 6-9; Denmark. 2013.

3. Sarai Mendoza-Armenta, Ian Dobson. Applying a formula for generator redispatch to damp interarea oscillations using synchrophasors. IEEE Transactions on Power Systems. 2013;31(4):3119-3128.

4. Madhvi Gupta, Vivek Kumar, Gopal Krishna Banerjee, et al. Mitigating congestion in a power system and role of facts devices. Advances in Electrical Engineering. 2017;2017:1-7. 